\title{
Leakage currents in high-permittivity thin films
}

\author{
Herbert Schroeder, ${ }^{\text {a) }}$ Sam Schmitz, and Paul Meuffels \\ Institut für Elektrokeramische Materialien im Institut für Festkörperforschung, Forschungszentrum Jülich \\ GmbH, D-52425 Jülich, Germany
}

(Received 20 August 2002; accepted 4 December 2002)

\begin{abstract}
Quite often leakage current data through high-permittivity thin films exhibit straight lines in the "Schottky" plot, i.e., log (current density $j$ ) versus sqrt (mean applied field), which suggests an electrode-limited current by field-enhanced thermionic emission. Unfortunately, the extracted permittivity at optical frequencies seldom is in agreement with experimental values and often is unacceptably small, i.e., $<1$. We suggest a model demonstrating that the leakage current in high-permittivity thin films is bulk-limited, but still is showing the characteristic dependence of thermionic emission. This is due to a combination of boundary conditions of the model, low-permittivity thin layers ("dead layer") at the electrodes and current injection/recombination terms at the injecting/collecting electrodes, respectively. (C) 2003 American Institute of Physics. [DOI: $10.1063 / 1.1541096]$
\end{abstract}

The steady state charge transport, i.e., the leakage current, through thin films of high permittivity materials such as $\mathrm{SrTiO}_{3}$ (STO) and $(\mathrm{Ba}, \mathrm{Sr}) \mathrm{TiO}_{3}(\mathrm{BST})$ is not very well understood. As the latter material is a serious candidate for use as dielectric in the capacitor of future Gb-generation dynamic random access memory cells, ${ }^{1}$ the understanding of the charge loss mechanisms in these materials, e.g., by leakage, has also an important technological aspect. There are mainly two groups of mechanisms under discussion: Injection (interface) limited currents or film bulk-limited currents. For the first, the most important mechanism under consideration is thermionic emission, possibly combined with tunneling. ${ }^{2-4}$ For the second group, mainly space charge limited current, Poole-Frenkel, and hopping conduction mechanisms are discussed. ${ }^{5,6}$ The basics for all these mechanisms can be found in various textbooks. ${ }^{7,8}$

The most favored mechanism is the thermionic emission which is described by Eq. (1) including the barrier reduction $\Delta \Phi$ (called Schottky effect) due to the combined effect of the electric field at the injecting interface, i.e., $E(x \approx 0)$, and the coulomb mirror potential of the traveling charges

$$
\begin{aligned}
& j=A^{*} T^{2} \exp \left[-\left(\Phi_{0}-\Delta \Phi\right) / k T\right] ; \\
& \Delta \Phi=\sqrt{e_{0}^{3} E(x \approx 0) / 4 \pi \varepsilon_{0} \varepsilon_{r, \mathrm{opt}}} .
\end{aligned}
$$

$j$ is the saturation current density, $A^{*}$ is the effective Richardson constant, i.e., the free electron value of $120 \mathrm{~A} / \mathrm{cm}^{2} \mathrm{~K}^{2}$ corrected by the effective electron mass (quantummechanical reflections are neglected), $T$ is the absolute temperature, $k$ is the Boltzmann constant, $\Phi_{0}$ is the zero field equilibrium barrier height, $e_{0}$ is the elementary charge, $\varepsilon_{0}$ is the vacuum dielectric constant, and $\varepsilon_{r \text {,opt }}$ is the relative dielectric constant at optical frequencies (about 5.6 for STO and BST), respectively. The two main reasons to favor that mechanism are: (i) The activation energies extracted from the temperature dependence of leakage current data are often consistent with the rule of thumb that $\Phi_{0}$ is the difference of the vacuum work function of the injecting electrode and the electron affinity of the dielectric. (ii) The field dependence of

${ }^{a}$ Electronic mail: he.schroeder@fz-juelich.de these data often shows straight $\operatorname{lines}$ if $\log j$ is plotted versus sqrt (applied field $E=U_{\text {ext }} / t$ ). $U_{\text {ext }}$ is the applied voltage, $t$ is the thickness of the dielectric film. Unfortunately, the evaluation of $\varepsilon_{r, \text { opt }}$ from the slope of these curves quite often results in unphysical values $<1 .{ }^{9}$ This can be healed by assuming a thin, low permittivity layer (the "dead layer") at the injecting interface $\left(\varepsilon_{I} \ll \varepsilon_{\text {film }}\right)$ as suggested by Zhou and Newns ${ }^{10}$ which increases $E(x \approx 0)$ roughly by the factor $\varepsilon_{\text {film }} / \varepsilon_{I},{ }^{4,11}$ Nevertheless, the identification of thermionic emission as the limiting mechanism for the current through the dielectric implies that this current can be transported by the film bulk to the collecting electrode without significant disturbance. As titanate based high permittivity materials such as STO or BST have very low electronic mobility, $\mu$, $\left(\approx 1 \mathrm{~cm}^{2} / \mathrm{V} \mathrm{s} \text { at } \mathrm{RT}\right)^{12}$ indicating strong interaction of the electronic carriers with the lattice, the interpretation of the leakage currents as electrode limited is questionable.

Therefore, the aim of the present work was to study the (band) conductivity of the dielectric film in a onedimensional model under the boundary condition of thermionic emission (including the Schottky effect) at the injecting electrode. We have restricted our model and the subsequent simulation studies to such conditions that the electron current always describes the total current, implying negligible hole current and no ionic contribution. The possible ionic defects are assumed to be immobile. The model used is schematically shown in Fig. 1. The dielectric of thickness $t$ consists of three layers: The high permittivity film (thickness $t-2 a$; permittivity $\varepsilon_{\text {film }}$ ) and a thin layer at each electrode $(M) /$ dielectric interface of thickness $a \ll t$ with a low permittivity $\varepsilon_{I} \ll \varepsilon_{\text {film }}$ (for convenience, the identical layers are assumed). All other properties (band gap energy, mobility, defect densities, etc.) are identical throughout the film. It is described as a linear dielectric, and symmetrical electrodes are assumed. All these restrictions reduce the complexity of the simulation calculation, but they can be dropped without significant change of the model.

As usual, within the dielectric, both the Poisson Eq. (2) and the continuity Eq. (3) have to be solved in all three regions of different $\varepsilon_{r}$ : 


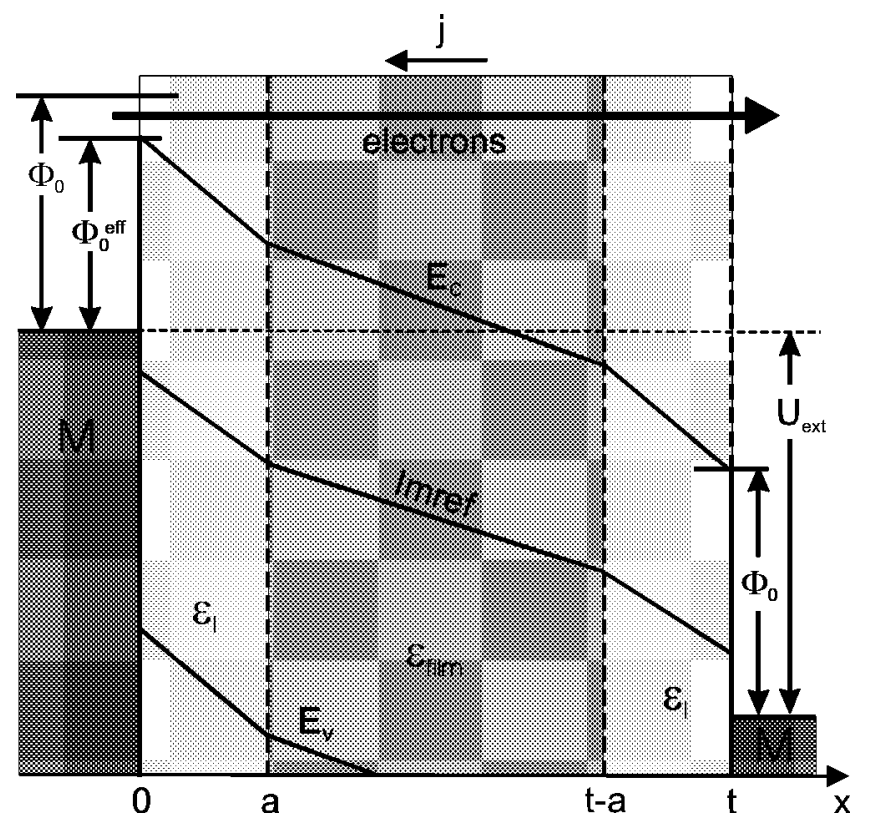

FIG. 1. Schematic band diagram of the metal-insulator-metal capacitor structure used for the model and the simulation calculations.

$$
\begin{aligned}
& \frac{\partial D(x)}{\partial x}=\frac{\varepsilon_{r} \quad \varepsilon_{0} \partial E(x)}{\partial x}=-\frac{\varepsilon_{r} \quad \varepsilon_{0} \partial^{2} \varphi(x)}{\partial x^{2}}=\rho(x) \\
& j=j_{n}=e_{0} n(x) \mu E(x)+k \quad T \mu \quad \partial n(x) / \partial x=\text { const, }
\end{aligned}
$$$$
n(x)=N_{C} \exp \left\{-\left[E_{C}(x)-E_{F}(x)\right] / k \quad T\right\} .
$$

$\rho(x)$ is the sum of possible electronic and ionic space charge densities, $\varphi(x)$ is the electric potential, $n(x)$ is the electron density, $N_{\mathrm{C}}$ is the effective density of states in the conduction band, $E_{\mathrm{C}}(x)$ and $E_{\mathrm{F}}(x)$ are the conduction band and Fermi energies, respectively. For the internal interfaces dead layer/ dielectric film at $x=a$ and $x=t-a$, the steadiness of the dielectric displacement, $D(x)$, results in jumps of the electrical field $E(x)[=-\partial \varphi(x) / \partial x]$ at these positions proportional to the ratio of the permittivities, indicated by the different slopes of the energy bands in Fig. 1, while the electric potential $\varphi(x)$ is steady. [Note: As usual, the electric potential energy of an electron is related to the conduction band energy, $E_{\mathrm{C}}(x)$, by $E_{\mathrm{C}}(x)=-e_{0} \varphi(x)$, except a constant.] As the electron density $n(x)$ is thus a function of $\varphi(x)$ [see Eq. (4)], it is steady, while its derivative, $\partial n(x) / \partial x$, has jumps at these positions, too.

The necessary boundary conditions have to be specified. Two boundary conditions are for the electric potential energy at the metal interfaces, i.e., the barrier heights at the electrodes

$$
\begin{aligned}
& E_{C}(x=0)=-e_{0} \varphi(0)=E_{\mathrm{FM} \mathrm{left}}+\Phi_{B, \text { left }} ; \\
& E_{C}(x=t)=-e_{0} \varphi(t)=E_{\mathrm{FM} \text { right }}+\Phi_{B, \text { right }} .
\end{aligned}
$$

Integration of Eq. (3) leads to the third boundary condition, connecting an externally applied voltage, $U_{\text {ext }}$, defined by the difference of the Fermi levels in the electrodes, i.e., $e_{0} U_{\text {ext }}=E_{\mathrm{FM} \text { right }}-E_{\mathrm{FM} \text { left }}$, to the potential drop (integral of the electrical field) inside the dielectric and a possible diffusion potential. $\sigma(x)=e_{0} n(x) \mu$ is the electrical conductivity Downloaded 21 Dec 2006 to 134.94.122.39. Redistribution subject

$$
\begin{aligned}
U_{e x t} & =\int_{0}^{t} d x \frac{j}{\sigma(x)}=\int_{0}^{t} d x\left[E(x)+\frac{k T}{e_{0}} \frac{\partial \ln n(x)}{\partial x}\right] \\
& =\varphi(0)-\varphi(t)+\frac{k T}{e_{0}} \ln \frac{n_{0}(t)}{n_{0}(0)} .
\end{aligned}
$$

Other "boundary" conditions are needed to describe the (electron) carrier injection (subscript $i$ ) from one metal electrode into the dielectric and the carrier collection (i.e., recombination, subscript $r$ ) at the other electrode, respectively. The recombination velocity approach $\left(v_{i, r}\right)$ suggested by Crowell and $\mathrm{Sze}^{13}$ for forward currents in metalsemiconductor Schottky barriers (diodes) and refined by Crowell and Beguwala ${ }^{14}$ also for the reversed direction (i.e., injection from the metal) will be used. They describe the (net) current density, $j_{i, r}$ at the interface as

$$
\begin{aligned}
& j_{i, r}=e_{0}\left[n\left(x_{m}\right)-n_{0}\right]_{i, r} \quad v_{i, r}, \\
& v_{i, r}=A^{*} T^{2} / e_{0} N_{C} .
\end{aligned}
$$

The position $x_{\mathrm{m}}(\approx 0)$ is defined by the energy potential maximum at the barrier, $n\left(x_{\mathrm{m}}\right)$ is the electron density at $x_{\mathrm{m}}$, when a net current is flowing, while $n_{0}$ is the quasiequilibrium electron density ${ }^{13}$ at $x_{\mathrm{m}}$. For a Maxwellian distribution of the electron velocities in the semiconductor, $v_{i, r}$ is given by Eq. (7b). For zero applied voltage the current is-of course-zero, i.e., $n\left(x_{\mathrm{m}}\right)=n_{0}$. This situation is the equilibrium ("Schottky") case, i.e., a steady Fermi energy at the interface. However, a net current, i.e., $n\left(x_{\mathrm{m}}\right) \neq n_{0}$, leads to a nonequilibrium Fermi energy, called Imref, if $n\left(x_{\mathrm{m}}\right)$ is described by Eq. (4). As $j<0$ for electrons (the electrons move into the opposite direction, see Fig. 1), an electron injection at the left metal electrode into the dielectric calls for $n\left(x_{\mathrm{m}}\right)$ $<n_{0}$ and Imref $\left[n\left(x_{\mathrm{m}}\right)\right]>E_{\mathrm{F}}\left(n_{0}\right)$ as indicated in Fig. 1. For the collecting right electrode it is the opposite, $n\left(\mathrm{t}-\mathrm{x}_{\mathrm{m}}\right)$ $>n_{0}$ and Imref $\left[n\left(\mathrm{t}-\mathrm{x}_{\mathrm{m}}\right)\right]<E_{\mathrm{F}}\left(n_{0}\right)$. As the injection current density, $j_{\mathrm{i}}$, and the recombination current density, $j_{\mathrm{r}}$, have to be equal to the current density inside the dielectric [Eq. (3)] because of $\operatorname{div} j=0$, the electron densities $n\left(x_{\mathrm{m}}\right)$ and $n\left(\mathrm{t}-\mathrm{x}_{\mathrm{m}}\right)$ (and thus the Imrefs at these positions) have to be adjusted consistently.

Equation (7a) can also be interpreted as the difference of two (particle) currents: One from the dielectric into the metal, described by $e_{0} n\left(x_{\mathrm{m}}\right) v$, the other from the electrode into the dielectric, $e_{0} n_{0} v$, which is equal to thermionic emission current [Eq. (1)] over the equilibrium barrier associated with $n_{0}$. Taking into account the image potential at both electrode interfaces leads to different barrier reductions, $\Delta \Phi$, at positions $x_{\mathrm{m}}$ and $\mathrm{t}-\mathrm{x}_{\mathrm{m}}$ and therefore different quasiequilibrium electron densities at these positions. This results in a nonzero diffusion potential [see Eq. (6)].

It is also worth noting that within this model the (electron) current density is always smaller than that predicted by the thermionic emission equation, so that Eq. (1) has to be regarded as an upper limit as long as tunneling contributions are neglected.

The differential Eq. (2) and (3) cannot be solved analytically for the general case. ${ }^{13}$ Therefore, computer calculations using the finite difference method ${ }^{15}$ with 200 slabs and the "Gauss-Seidel" iteration method have been performed. Many external and internal parameters have been varied to AIP license or copyright, see http://apl.aip.org/apl/copyright.jsp 


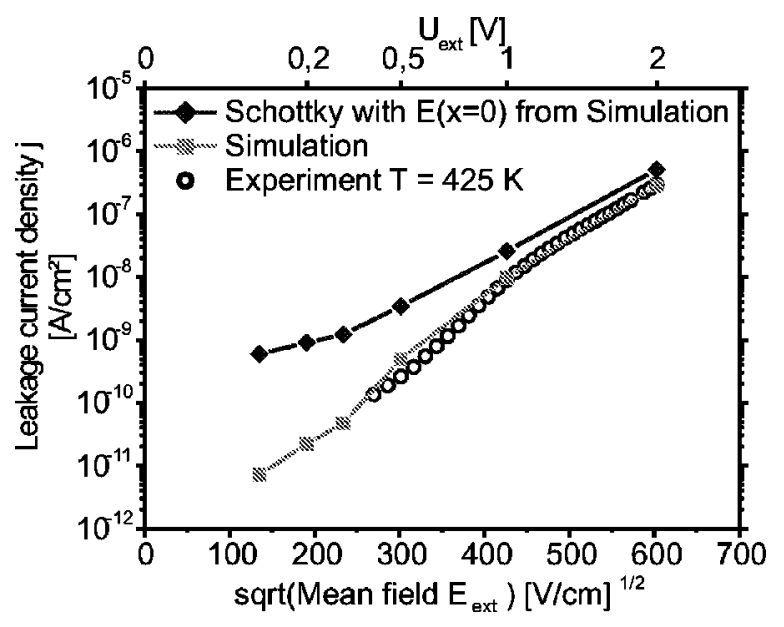

FIG. 2. Comparison of leakage current densities: (○) Experiment; ( $\square$ ) finite difference method simulation calculation; and $(\bullet)$ thermionic emission.

study their implications on the leakage current within this model, e.g., temperature, applied field, dielectric thickness, donor concentration, thickness and permittivity of the dead layers, electron mobility, diffusivity, zero field barrier height (simulating different electrodes). Details of these simulation calculations will be reported in a subsequent full paper. Instead, some important trends will be mentioned and an example for a fit of a simulation to experimental leakage current data will be shown.

The comparison between simulation and experimental data is shown in Fig. 2 as a Schottky plot, i.e., $\log j$ vs sqrt $\left(U_{\text {ext }} / t\right)$ : The open circles are experimental leakage data ${ }^{16}$ from a $55 \mathrm{~nm}$ thick BST sample between Pt electrodes measured at $425 \mathrm{~K}$. The BST was produced by metalorganic chemical vapor deposition at a substrate temperature of 870 $\mathrm{K}$, the ratio $\mathrm{Ba}: \mathrm{Sr}$ was 70:30, and it was Ti-rich (ratio Ti: $(\mathrm{Ba}+\mathrm{Sr})=1.05) .{ }^{17}$ At high fields the data follow a straight line, quite often interpreted as an indication for electrode limited current due to thermionic emission, while at low fields some deviations from this behavior are seen. The full squares represent the "best" fit (from the calculations done up to now). Besides the given external parameters (measuring temperature, applied mean field, BST thickness) other parameters used as input were determined from separate measurements: The electron mobility $\mu_{\mathrm{n}}=0.74 \mathrm{~cm}^{2} / \mathrm{V} \mathrm{s}$ (connected to the diffusivity by the Einstein relation: $e_{0} D_{\mathrm{n}}$ $=k T \mu_{\mathrm{n}}$ ) from single crystal measurement in the literature, ${ }^{12}$ permittivity of the film bulk, $\varepsilon_{\text {film }}=550$, and the ratio $\varepsilon_{I} / a$ $=11$ from the thickness dependence of the capacitance evaluated with the three layer model. ${ }^{16}$ Therefore, only very few parameters were adjusted: Choosing the dead layer thickness $a=1.1 \mathrm{~nm}$ results in $\varepsilon_{I}=12$; the zero field barrier height was set to $1.52 \mathrm{eV}$ and the donor density to $10^{18} \mathrm{~cm}^{-3}$ with a donor energy level close to the conduction band. The free electron mass was used, thus keeping $N_{\mathrm{C}}$ and $A^{*}$ to their free electron values. With this parameter set a nearly perfect fit to the experimental data was achieved. It should be noted that a similar result was possible with slightly changed parameters if some variation in the values extracted from measurements was allowed.

The adjusted parameters are reasonable: The dead layer thickness is in the range of $1-3 \mathrm{~nm}$ as proposed by a theo- retical model. ${ }^{10}$ Assuming $4.1 \mathrm{eV}$ for the BST electron affinity ${ }^{18}$ the barrier height $\Phi_{0}=1.52 \mathrm{eV}$ leads to a vacuum work function for Pt of $5.6 \mathrm{eV}$ (using the mentioned rule of thumb) close to the values in the literature. ${ }^{8,18}$ A donor concentration of $10^{18} \mathrm{~cm}^{-3}$, e.g., oxygen vacancies, is not unusual for thin films.

The full diamonds in Fig. 2 represent the thermionic emission saturation current density, Eq. (1), using the electric field at the injecting electrode from the simulation calculation. As expected, it is always larger than the simulated current, at high applied fields about a factor of 2, at low fields up to two orders of magnitude. This clearly demonstrates that the leakage current in low-mobility, high-permittivity dielectrics is bulk limited even in very thin films. In contrast, if the mobility is increased by two orders of magnitude, representing common semiconductors such as $\mathrm{Si}$ or Ge, the simulated currents are approaching the thermionic emission limit in the whole field range rather closely.

As mentioned tunneling contributions, which should be most important at lower temperatures and higher fields, have not been included in the simulation calculations. In contrast, in Refs. 3, 4, and 11 tunneling was considered by using the combined injection currents over the barrier (thermionic emission) and through the barrier (tunneling). In these works always the saturation limits of these injection currents (except in Ref. 11 for the thermionic emission part) were used for the calculations and comparisons with experimental data. This contradicts the main result of the presented model that these limits are hardly reached in low mobility insulators. As there is no general difference if the electron is injected over or through the barrier into the insulator an injection term for tunneling equivalent to Eq. (7a) should be used. The corresponding velocity in this term will have-of coursedifferent temperature and field dependencies adjusted to the saturation current of the tunneling contribution.

${ }^{1}$ D. E. Kotecki, J. D. Baniecki, H. Shen, R. B. Laibowitz, K. L. Saenger, J. J. Lian, T. M. Shaw, S. D. Athavale, C. Cabral, P. R. Duncombe, M. Gutsche, G. Kunkel, Y.-J. Park, Y.-Y. Wang, and R. Wise, IBM J. Res. Dev. 43, 367 (1999)

${ }^{2}$ G. W. Dietz, M. Schumacher, R. Waser, S. K. Streiffer, C. Basceri, and A. I. Kingon, J. Appl. Phys. 82, 2359 (1997).

${ }^{3}$ G. W. Dietz and R. Waser, Thin Solid Films 299, 53 (1997).

${ }^{4}$ J. C. Shin, J. Park, C. S. Hwang, and H. J. Kim, J. Appl. Phys. 86, 506 (1999).

${ }^{5}$ K. Watanabe, A. J. Hartmann, R. N. Lamb, and J. F. Scott, Integr. Ferroelectr. 21, 241 (1998).

${ }^{6}$ S. Saha and S. B. Krupanidhi, Appl. Phys. Lett. 79, 111 (2001).

${ }^{7}$ J. J. O'Dwyer, The Theory of Electrical Conduction and Breakdown in Solid Dielectrics (Clarendon, Oxford, 1973).

${ }^{8}$ S. M. Sze, Physics of Semiconductor Devices, 2nd ed. (Wiley, New York, 1981).

${ }^{9}$ S. Zafar, R. E. Jones, B. Jiang, B. White, V. Kaushik, and S. Gillespie, Appl. Phys. Lett. 73, 3533 (1998).

${ }^{10}$ C. Zhou and D. W. Newns, J. Appl. Phys. 82, 3081 (1997).

${ }^{11}$ J. D. Baniecki, R. B. Laibowitz, T. M. Shaw, C. Parks, J. Lian, H. Xu, and Q. Y. Ma, J. Appl. Phys. 89, 2873 (2001).

${ }^{12}$ I. Denk, W. Münch, and J. Maier, J. Am. Ceram. Soc. 78, 3265 (1995).

${ }^{13}$ C. R. Crowell and S. M. Sze, Solid-State Electron. 9, 1035 (1966).

${ }^{14}$ C. R. Crowell and M. Beguwala, Solid-State Electron. 14, 1149 (1971).

${ }^{15}$ T. R. Brumleve and R. P. Buck, J. Electroanal. Chem. 90, 1 (1978).

${ }^{16}$ S. Schmitz, Thesis, RWTH Aachen (Germany), 2002; Reports of the Research Center Jülich GmbH, JÜL-4006, October 2002.

${ }^{17}$ P. Ehrhart, F. Fitsilis, S. Regnery, C. L. Jia, H. Z. Jin, R. Waser, F. Schienle, M. Schumacher, and H. Juergensen, Mater. Res. Soc. Symp. Proc. 655, CC9.4.1 (2001).

${ }^{18}$ J. F. Scott, Jpn. J. Appl. Phys., Part 1 38, 2272 (1999). 